OPEN ACCESS

Edited by:

Alessandro Tozzi,

University of Perugia, Italy

Reviewed by:

Lachlan Thompson,

University of Melbourne, Australia

Cesar V. Borlongan,

University of South Florida,

United States

*Correspondence: Hans R. Widmer hanswi@insel.ch

Received: 15 February 2017 Accepted: 09 May 2017 Published: 26 May 2017

Citation:

Seiler S, Di Santo S, Andereggen L and Widmer HR (2017) Antagonization of the Nogo-Receptor 1 Enhances Dopaminergic Fiber

Outgrowth of Transplants in a Rat Model of Parkinson's Disease. Front. Cell. Neurosci. 11:151. doi: 10.3389/fncel.2017.00151

\section{Antagonization of the Nogo-Receptor 1 Enhances Dopaminergic Fiber Outgrowth of Transplants in a Rat Model of Parkinson's Disease}

\author{
Stefanie Seiler ${ }^{1,2}$, Stefano Di Santo 1,2, Lukas Andereggen ${ }^{1,2}$ and Hans R. Widmer ${ }^{1,2 *}$ \\ ${ }^{1}$ Department of Neurosurgery, Neurocenter and Regenerative Neuroscience Cluster, University Hospital Bern, Switzerland \\ University of Bern, Bern, Switzerland, ${ }^{2}$ Department of Clinical Research, University of Bern, Bern, Switzerland
}

Intrastriatal transplantation of fetal human ventral mesencephalic dopaminergic neurons is an experimental therapy for patients suffering from Parkinson's disease. The success of this approach depends on several host brain parameters including neurotrophic factors and growth inhibitors that guide survival and integration of transplanted neurons. While the potential of neurotrophic factors has been extensively investigated, repression of growth inhibitors has been neglected, despite the significant effects reported in various CNS injury models. Recently, we demonstrated that infusion of neutralizing antibodies against Nogo-A into the lateral ventricles of hemi-parkinsonian rats significantly enhanced graft function. Since the Nogo-receptor 1 also interacts with other neurite growth inhibitors, we investigated whether a direct antagonization of the receptor would result in more robust effects. Therefore, rats with unilateral striatal 6-hydroxydopamine lesions were grafted with ventral mesencephalic tissue in combination with intraventricular infusions of the Nogo-receptor 1 antagonist NEP1-40. Transplanted rats receiving saline infusions served as controls. To test whether NEP1-40 treatment alone affects the remaining dopaminergic striatal fibers, rats with unilateral striatal 6-hydroxydopamine lesions were infused with NEP1-40 or saline without receiving a transplant. Motor behavior was assessed prior to the lesion as well as prior and 1, 3, and 5 weeks after the transplantations. At the end of the experimental period the number of graft-derived dopaminergic fibers growing into the host brain, the number of surviving dopaminergic neurons and graft volume were analyzed. In rats without a transplant, the density of dopaminergic fibers in the striatum was analyzed. We detected that NEP1-40 treatment significantly enhanced graft-derived dopaminergic fiber outgrowth as compared to controls while no effects were detected for graft volume and survival of grafted dopaminergic neurons. Notably, the enhanced dopaminergic fiber outgrowth was not sufficient to improve the functional recovery as compared to controls. Moreover, NEP1-40 infusions in hemi-parkinsonian rats without a transplant 
did not result in enhanced striatal dopaminergic fiber densities and consequently did not improve behavior. In sum, our findings demonstrate that antagonization of the Nogo-receptor 1 has the capacity to support the engraftment of transplanted mesencephalic tissue in an animal model of Parkinson's disease.

Keywords: Parkinson's disease, Nogo-receptor 1, cell transplantation, dopaminergic neurons, behavior, rat

\section{INTRODUCTION}

Parkinson's disease (PD) is a neurodegenerative disease mainly characterized by progressively degenerating dopaminergic neurons in the substantia nigra pars compacta. The resulting lack of dopaminergic innervation of the striatum and consequent loss of dopamine leads to severe motor symptoms. Cell replacement strategies, i.e., transplantation of human fetal ventral mesencephalic (VM) tissue into the caudate and putamen of patients, that intend to restore the lost dopamine in the striatum, can result in considerable long-term motor improvement up to several years after transplantation (Yasuhara et al., 2006; Hallett et al., 2014; Kefalopoulou et al., 2014; Pantcheva et al., 2015) and are thus a promising experimental approach to treat PD (Bjorklund et al., 2003; Ourednik and Ourednik, 2005; Piquet et al., 2012). For a successful outcome, it is important to assure the survival of grafted dopaminergic cells and their functional integration into the host brain (Emgard et al., 1999; Karlsson et al., 2000; Rath et al., 2013), a process in which the host microenvironment plays a crucial role (Ourednik and Ourednik, 2005; Alsberg et al., 2006; Stefanova et al., 2009; Akbik et al., 2012). The microenvironment consists of the totality of signals induced by local neurotrophic and neurite growth inhibiting factors. The significant improvement in engraftment of VM tissue by administering neurotrophic factors, such as glial cell line-derived neurotrophic factor, brain-derived neurotrophic factor, and neurotrophin $4 / 5$ into the host striatum has been demonstrated years ago (Haque et al., 1996; Rosenblad et al., 1996; Sinclair et al., 1996; Yurek et al., 1996; Yurek, 1998; Espejo et al., 2000; Chaturvedi et al., 2006; Tatard et al., 2007; Thompson et al., 2009). While inhibition of Nogo-A signaling, one of the most potent neurite growth inhibitor in the central nervous system (CNS), has been successfully implemented in various models of CNS injuries (Gonzenbach and Schwab, 2008; Schwab and Strittmatter, 2014), it has so far been largely neglected in studies of cell transplantation approaches for PD. We have recently demonstrated that intraventricular infusion of neutralizing anti-Nogo-A antibodies enhanced survival of grafted dopaminergic neurons and significantly promoted their fiber outgrowth, resulting in an improved functional recovery of the hemi-parkinsonian rats (Seiler et al., 2016). Nogo-A can bind to the Nogo-receptor 1 (NgR1), which then interacts with the leucine rich repeat neuronal protein (LINGO-1) and the low affinity nerve growth factor receptor (p75) and/or the tumor necrosis family member LIMK (TROY) before activating the Rho/ROCK pathway (Schmandke et al., 2014). We and others have shown, that both the neutralization of Nogo-A and the antagonization of the NgR1 complex improve the survival and morphology of dopaminergic neurons. In fact, deletion or antagonization of LINGO-1 enhanced the survival of dopaminergic neurons in an in vitro and mouse model of PD (Inoue et al., 2007). Moreover, we could show that antagonization of NgR1 by the peptide NEP1-40 significantly improved the survival of dopaminergic neurons and their morphological complexity in fetal primary VM cultures (Seiler et al., 2013). Based on these observations, we aimed at investigating whether NgR1 antagonization by NEP1-40 improves survival and integration of grafted dopaminergic neurons and functional recovery in a hemi-parkinsonian rat model.

\section{MATERIALS AND METHODS}

\section{Animals}

Adult female Wistar rats (Janvierlabs, France) were housed at $12 \mathrm{~h}$ light dark cycle with food and water ad libitum. For the preparation of the transplants, time-pregnant Wistar dams were purchased from Janvierlabs (France). All experiments were carried out in the light phase and in accordance with the guidelines of the Animal Research Ethics Committee of the Canton Berne, Switzerland, and the University of Bern Animal Care and Use Committee, Switzerland.

\section{Experimental Design}

The experimental design is schematically depicted in Figure 1 and was planned similar to our previous study (Seiler et al., 2016). In the first experimental setup, the hemi-parkinsonian rats received a transplant and a mini-osmotic pump releasing either saline or NEP1-40 into the right lateral ventricles (Figure 1A). In the second experimental setup, hemi-parkinsonian rats received only mini-osmotic pumps releasing either saline or NEP1-40 into the right lateral ventricles (Figure 1B). To create hemi-parkinsonian rats, 6-hydroxydopamine (6-OHDA) was injected into the right striatum. Six week after the lesions, fetal rat VM tissue was transplanted into the right striatum. In the same surgical procedure, mini-osmotic pumps that continuously infused either saline or NEP1-40 into the right lateral ventricle were implanted (Figure 1A). Six weeks after the transplantations rats were perfused and their brains prepared for histological analyses. In these rats asymmetrical forelimb use was assessed by means of the cylinder test before (baseline) and 5 weeks after the lesions (lesioned) and 1,3, and 5 weeks after the transplantations (Figure 1A). In the rats implanted with mini-osmotic pumps only asymmetrical forelimb use was assessed with the cylinder test before the lesion (baseline), 5 weeks after the lesion (lesioned) and 5 weeks after the pump implantation (Figure 1B). 
A

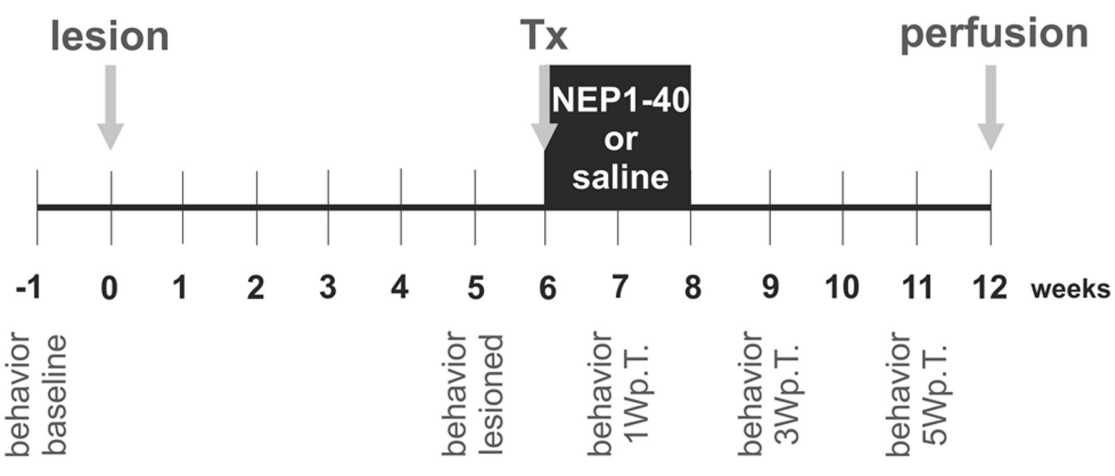

B

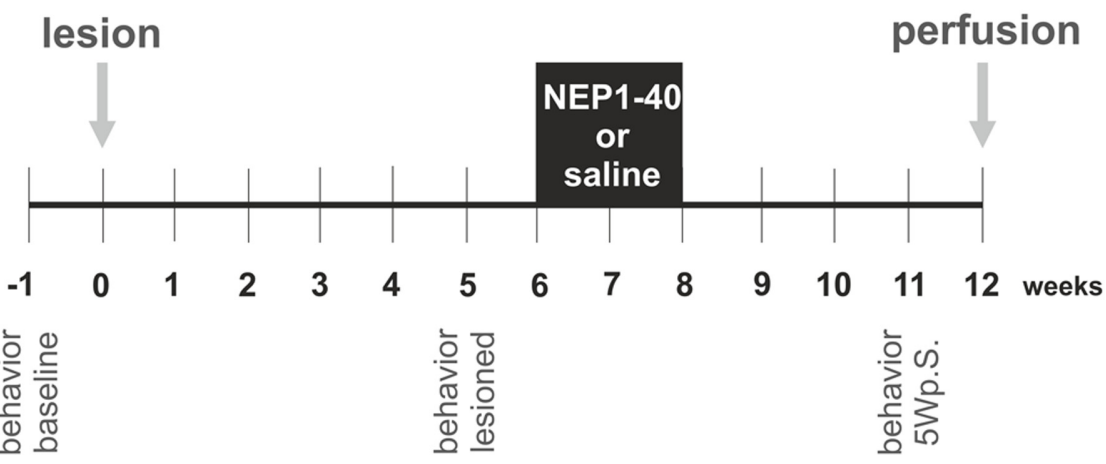

FIGURE 1 | Experimental design of the study. The experiments were conducted in two experimental set ups. In the first experiment hemi-parkinsonian rats received a transplant and a mini-osmotic pump (A) and in the second experiment hemi-parkinsonian rats received only a mini-osmotic pump and no transplant (B). The neurotoxin 6-hydroxydopamine was injected unilateral into the right striatum of rats (lesion) and 6 weeks later the rats of experimental setup 1 received a transplant of two ventral mesencephalic free-floating roller tube cultures ( $T x)$. Mini-osmotic pumps were implanted into all hemi-parkinsonian rats, releasing either saline or NEP1-40 over a period of 2 weeks into the lateral ventricles (black box). Six weeks after the surgeries rats were transcardially perfused and the brains removed for histological analyses. The cylinder test was assessed in the rats of both the experimental groups, 1 week before the lesion (behavior baseline) and 1 week prior to surgery (behavior lesioned). In the experimental setup 1 behavioral analyzes were performed 1, 3, and 5 weeks post transplantation (behavior 1Wp.T.; behavior 3Wp.T.; behavior 5Wp.T.). In the experimental setup 2 behavior was evaluated 5 weeks after the surgery (behavior 5Wp.S.).

\section{Parkinson's Disease Rat Model}

Female Wistar rats (200-270 g; Janvierlabs, France) were anesthetized with Isoflurane $\left(75 \% \mathrm{~N}_{2} \mathrm{O}, 20 \% \mathrm{O}_{2}, 4.5-5 \%\right)$ followed by an intraperitoneal (i.p.) injection of Narketan (75 mg/kg; Vétoquind AG, Ittigen, $\mathrm{CH}$ ) and Xylazine $(5 \mathrm{mg} / \mathrm{kg}$; Vétoquind AG, Ittigen, $\mathrm{CH})$. Buprenorphine $(0.5 \mathrm{mg} / \mathrm{kg}$; Reckitt Benckiser AG, Wallisellen) was subcutaneously (s.c.) injected $30 \mathrm{~min}$ before the surgical intervention. Under deep anesthesia the rats were placed on a heating pad and fixed in a stereoscopic frame (Stoelting Co.). Through a small burr hole in the skull $4 \mu \mathrm{l}$ of 6-OHDA (32 mM 6-OHDA; H116 Sigma-Aldrich Chemie $\mathrm{GmbH}$ ) were injected into the right striatum according to the following coordinates in relation to bregma (Paxinos Watson rat brain atlas): anterior $1.0 \mathrm{~mm}$, lateral $3.0 \mathrm{~mm}$ and $5.0 \mathrm{~mm}$ ventral to the dura, the incisor bar was set at $0.0 \mathrm{~mm}$. The injection rate was $1 \mu \mathrm{l} / \mathrm{min}$. The syringe was left in place for additional $4 \mathrm{~min}$ before slowly retracting the needle.

\section{Preparation of Transplants}

The tissues for the transplantations were organotypic fetal rat free-floating roller tube cultures prepared from embryonic day 14 (E14) VM as described previously (Andereggen et al., 2009; Seiler et al., 2016). In brief, time-pregnant Wistar rats were anesthetized with Isoflurane $\left(75 \% \mathrm{~N}_{2} \mathrm{O}, 20 \% \mathrm{O}_{2}, 4.5-5 \%\right)$ followed by an i.p. injection of Narketan $(120 \mathrm{mg} / \mathrm{kg})$ and Xylazine $(20 \mathrm{mg} / \mathrm{kg})$ and their fetuses removed by cesarean section. The VM was separated from the brain under a stereoscopic microscope and cut into four equally sized pieced that correspond to two rostral and two caudal portions. Each piece was placed into a gas-permeable conical plastic tube (Falcon) filled with $1 \mathrm{ml}$ of culture medium consisting of 55\% DMEM, 32.5\% Hank's balanced salt solution (HBSS; Gibco), $0.3 \%$ glucose, $10 \%$ fetal calf serum (FCS; Gibco) and $1 \% 0.01 \mathrm{M}$ HEPES (Merck) as well as antibiotics/antimycotics (No. 061-052 40 D; Gibco). Each tube was positioned in a rotating roller drum that was placed in an incubator at $37^{\circ} \mathrm{C}$ in a $5 \% \mathrm{CO}_{2}$ atmosphere as described in detail previously 
(Spenger et al., 1994). The VM tissue was grown for 7 days and the medium was changed after 2 and 5 days in vitro.

\section{Transplantation and Pump Implantation}

Six weeks after the 6-OHDA lesions, rats were anesthetized with Isoflurane $\left(75 \% \mathrm{~N}_{2} \mathrm{O}, 20 \% \mathrm{O}_{2}, 4.5-5 \%\right)$ followed by an i.p. injection of Narketan (75 mg/kg; Vétoquinol AG, Ittigen, $\mathrm{CH}$ ) and Xylazine (5 mg/kg; Vétoquinol AG, Ittigen, $\mathrm{CH})$. Buprenorphine ( $0.5 \mathrm{mg} / \mathrm{kg}$; Reckitt Benckiser AG, Wallisellen) was s.c. injected $30 \mathrm{~min}$ before the surgical intervention. In deep anesthesia the rats were put on a heating pad and mounted on a stereoscopic frame. Half a ventral mesencephalon consisting of one rostral and one caudal part of one embryo was transplanted into the right striatum. This guaranteed approximately equal amounts of dopaminergic neurons per graft. The following coordinates in relation to bregma (Paxinos Watson rat brain atlas) were used: anterior $1.0 \mathrm{~mm}$, lateral $2.7 \mathrm{~mm}$ and $4.5 \mathrm{~mm}$ ventral to the dura, the incisor bar was set at $0.0 \mathrm{~mm}$. Next, mini-osmotic pumps $\left(2 \mathrm{ml}^{2}\right.$, Alzet osmotic pumps, DURECT Corporation ALZET Osmotic Pumps) pre-filled with saline or NEP1-40 (75 $\mu \mathrm{g} / \mathrm{kg} /$ day) (GrandPre et al., 2002) were implanted under the skin of all the rats and the cannulas were subcutaneously connected to the skull and placed into the right ventricles (Alzet brain infusion kit2) according to the following coordinates in relation to bregma (Paxinos Watson rat brain atlas): posterior $0.8 \mathrm{~mm}$, lateral $1.6 \mathrm{~mm}$ and $3.5 \mathrm{~mm}$ ventral to the dura, the incisor bar was set at $0.0 \mathrm{~mm}$. The substances were administered by the miniosmotic pumps continuously over the subsequent 2 weeks with a flow rat of $5 \mu \mathrm{g} / \mathrm{h}$. The animals were randomly assigned to the two treatment groups (saline $n=6$; NEP1-40 $n=7$ ). Rats from the non-grafted groups (experimental setup 2) experienced the same mini-osmotic pump surgeries (saline $n=7$; NEP1-40, $n=7)$. Animals were let to recover for 1 week after the surgery.

\section{Behavior Test Series}

The cylinder test is a reliable measure to assess the asymmetry in forelimb use as observed after a unilateral lesion of the dopaminergic nigro-striatal pathway (Brooks and Dunnett, 2009; Schaar et al., 2010) and was evaluated as previously described (Seiler et al., 2016). In brief the rats were placed in a transparent cylinder (diameter $30 \mathrm{~cm}$ and height $41 \mathrm{~cm}$ ) with mirrors placed around it to allow a 360 degree view on the cylinder walls. The $10 \mathrm{~min}$ video recordings of the rat's behavior were analyzed by a researched blinded to the treatment groups by counting the number of wall touches with the left, the right and both paws together. To discriminate between a meaningful physiological movement and an accidental touch, only wall contacts by which the rat supported its body weight on the forelimb with extended digits were counted. One animal in the control group had to be excluded from the analysis as it did not touch the wall at all after the lesion. The percentage of left wall touches are calculated according to the formula: $[($ left $+1 / 2$ of both paw touches $) /($ left + right + both paw touches)] ${ }^{*} 100$ as previously described (Boix et al., 2015; Seiler et al., 2016).

\section{Perfusions}

Six weeks after the transplantation, rats were anesthetized with Isoflurane $\left(75 \% \mathrm{~N}_{2} \mathrm{O}, 20 \% \mathrm{O}_{2}, 4.5-5 \%\right)$ followed by an i.p. injection of Narketan (75 mg/kg) and Xylazine $(5 \mathrm{mg} / \mathrm{kg})$. Fentanyl (0.005 mg/kg, Janssen-AG, Zug, CH) was i.p. injected just prior to opening the thorax and the rats were perfused with $200 \mathrm{ml}$ ice cold 0.1 M phosphate buffer saline (PBS, pH 7.4) containing heparin (1000 I. E./100 ml, NOVO Nordisk) followed by $250 \mathrm{ml} \mathrm{4 \%}$ paraformaldehyde in $0.1 \mathrm{M}$ PBS. The brains were removed from the skull and placed in 4\% paraformaldehyde overnight and thereafter cryoprotected in 10\% sucrose-PBS solution.

\section{Immunohistochemistry}

Immunohistochemistry was performed as described previously (Seiler et al., 2016). The brains were cut at $30 \mu \mathrm{m}$ on a cryostat (Leica CM 1900) and mounted on Superfrost slides (Thermo Scientific). Sections were heated in citrate buffer for $30 \mathrm{~min}$ and blocked with $10 \%$ horse serum in $0.1 \%$ Triton-PBS. Primary and secondary antibodies were incubated in a $0.1 \%$ Triton-PBS solution containing $2.5 \%$ horse serum. Slides were incubated with the mouse monoclonal anti-tyrosine hydroxylase (TH; 1:1000, Millipore) overnight. After PBS washes, sections were incubated for $2 \mathrm{~h}$ with the secondary biotinylated anti-mouse IgG antibody (1:200, Vector Laboratories) and the endogenous peroxidase blocked with a solution of $10 \%$ methanol and 3\% hydrogen peroxide in PBS. Thereafter, the slides were incubated with an avidin-biotincomplex (7 $\mu \mathrm{l} / \mathrm{ml}$; Vectastain ABC-Peroxidase KIT, Vector Labs) for $1 \mathrm{~h}$ and specifically bound antibodies were visualized with a metal-enhanced 3,3'-diaminobenzidine substrate kit (Pierce, 34002, Life Technologies). The sections were dehydrated in alcohol, cleared in xylene and mounted in Eukitt (O. Kindler $\mathrm{GmbH}$, Freiburg, Germany).

\section{Histological Analyses}

All analyses were done by a researcher blinded to the treatment groups.

\section{Estimation of TH Positive Cells in the SNc}

The estimation of the extent of the lesion was done as described previously (Tronci et al., 2012; Seiler et al., 2016). In brief, brain sections of each rat were chosen that match the following coordinates in relation to bregma (Paxinos Watson rat brain atlas): posterior $4.8 \mathrm{~mm}$ (three sections analyzed, range $4.7-4.9 \mathrm{~mm}$ ), $5.3 \mathrm{~mm}$ (three sections analyzed, range 5.2-5.4 $\mathrm{mm}$ ), and $5.8 \mathrm{~mm}$ (three sections analyzed, range 5.7-5.9 mm). A light microscope equipped with a motorized stage and a video camera connected to a PC was used for counting. After delineation of the SNc with a $1 \times$ objective, the CAST system (Visiopharm) generated an unbiased counting frame with the $40 \times$ objective within the delineated SNc area. Only TH positive cells with a clearly stained cell body that were within the counting frame were counted. Data are expressed as percentage of $\mathrm{TH}$ positive cells on the lesioned side as compared to the number of $\mathrm{TH}$ positive neurons on the unlesioned side. 
A

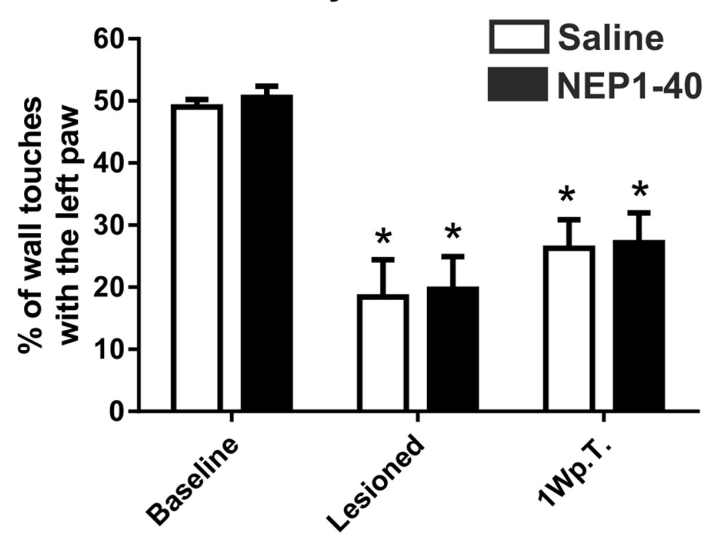

C

Cylinder Test

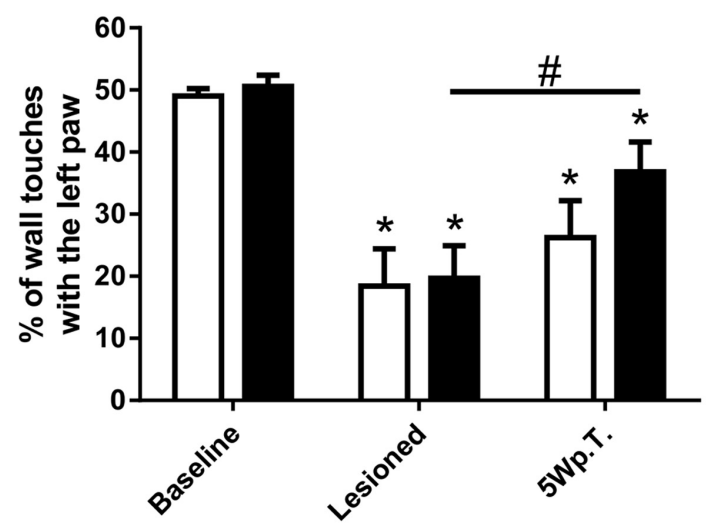

B

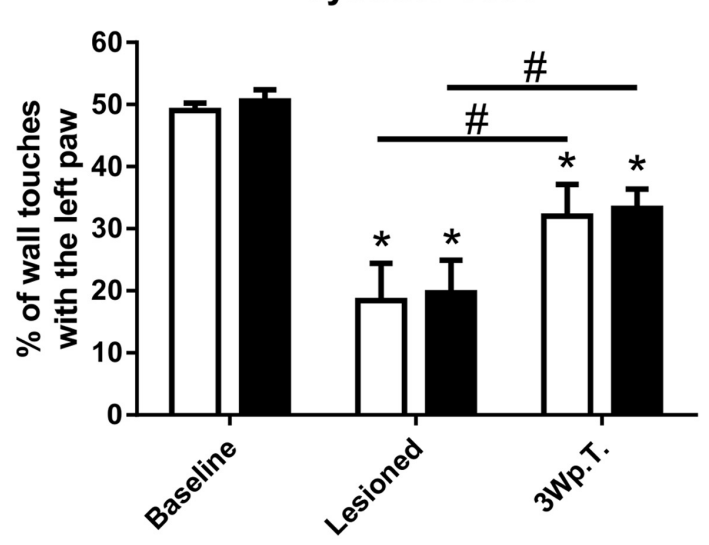

D

Cylinder Test

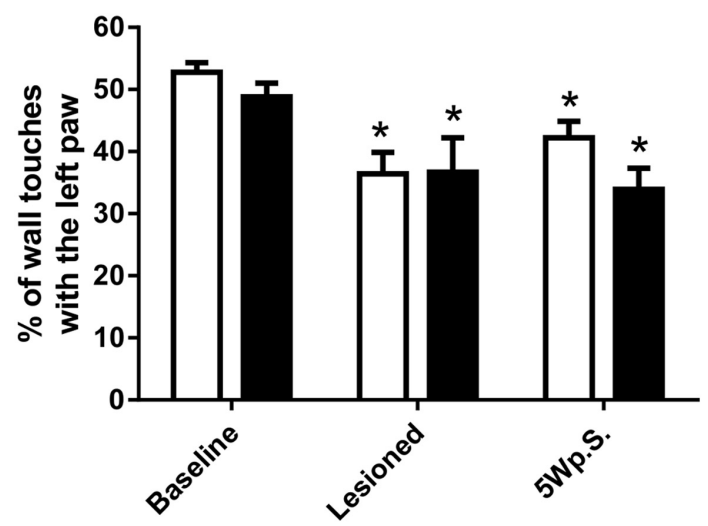

FIGURE 2 | Asymmetrical forelimb use of saline or NEP1-40 treated rats assessed with the cylinder test. The contralateral forelimb use (left paw use) is shown before (baseline) and after the 6-hydroxydopamine lesions as well as 1 (1Wp.T.; A), 3 (3Wp.T.; B) and 5 weeks post transplantation (5Wp.T.; C) in the experimental setup 1. The lesions resulted in a significantly reduced number of wall touches with the left paw as compared to baseline levels (A-C). Animals exposed to NEP1-40 treatment gradually improved the asymmetrical forelimb use over the 5 weeks period (B,C), however, did not reach baseline level (A-C). While an overall similar outcome was observed for the saline treated group at 5Wp.T. these animals did not perform significantly better than at the post-lesion time point (C). In the experimental setup 2, no behavioral recovery was found 5 weeks after pump implantation (5Wp.S) in both groups (D). Data are given as mean + SEM and expressed as percentage of left paw use. ${ }^{*} p<0.05$ vs. corresponding baselines, ${ }^{\#} p<0.05$ vs. corresponding lesion levels.

\section{Estimation of the Graft Volume}

Every third section containing a graft was selected to determine the size of the graft as previously reported (Andereggen et al., 2009; Seiler et al., 2016). In brief, an Olympus microscope (Olympus DP72) equipped a digital camera and connected to a PC with a calibrated neuron tracing software (CellSens Dimension, Olympus) was used to trace the graft boundaries. Thereafter, an automated computation integrated the areas to yield the graft volume. Graft volume in control animals was $0.21 \pm 0.03 \mathrm{~mm}^{3}$ (mean $\left.\pm \mathrm{SEM} ; n=6\right)$.

\section{Estimation of the TH Positive Fibers Growing into the Host Brain \\ Every third section containing a graft was selected to determine the number of $\mathrm{TH}$ positive fibers growing $100 \mu \mathrm{m}$ into the host brain as previously described (Seiler et al., 2016). In brief, an}

Olympus microscope (Olympus DP72) equipped a digital camera and connected to a PC with a calibrated neuron tracing software (CellSens Dimension, Olympus) was used to draw a virtual line $100 \mu \mathrm{m}$ from the graft boundary with a length of $300 \mu \mathrm{m}$. TH positive fibers originating in the graft were followed up to this virtual line and counted if they crossed this line. The counts were done at four sites, i.e., medial, lateral, dorsal, and ventral from the boarder of the graft using a $10 \times$ objective and verified with a $40 \times$ objective. Number of TH positive fibers in control animals were $5.5 \pm 1.1,7.7 \pm 0.4,4.6 \pm 0.9$, and $5.8 \pm 1.1$ crossing the virtual line at the medial, lateral, dorsal and ventral sites of the graft, respectively (mean \pm SEM; $n=6$ ).

\section{Estimation of the TH Positive Cells in the Graft}

Every third section containing a graft was selected to determine the number of $\mathrm{TH}$ positive cells in the graft using the optical 

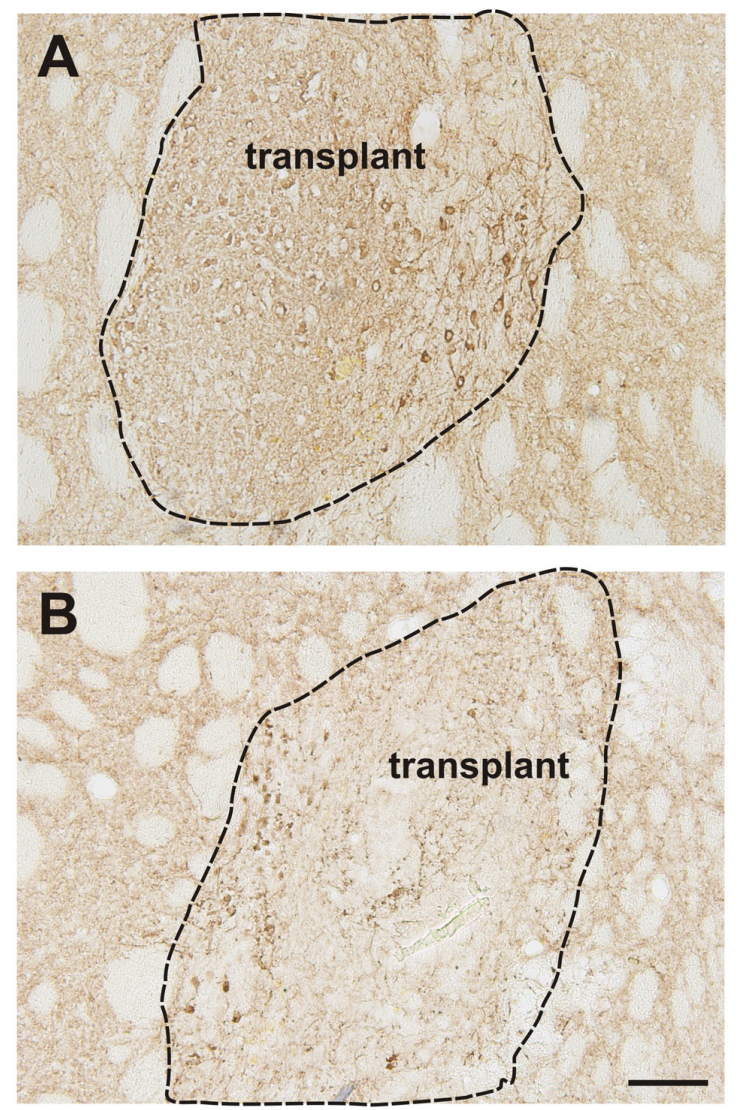

C

Graft volume

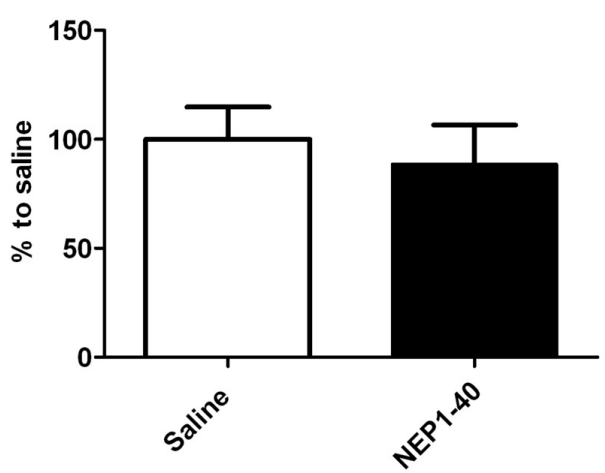

FIGURE 3 | Effects of intra-ventricular infusion of saline or NEP1-40 on graft volumes. Representative photomicrographs of intrastriatal grafts treated with either saline (A) or NEP1-40 (B) stained for tyrosine hydroxylase. The graft boarder is highlighted with a dashed black line. Scale bar: $100 \mu \mathrm{m}$. No difference in graft volume was detected between the two treatment groups, as shown in the bar graph (C). Data are given as mean + SEM and are presented as percentage of saline.

fractionators design as described by West et al. (1991), Kelsen et al. (2010). An Olympus light microscope (Olympus DP72) equipped with a motorized stage and a digital camera connected to a PC was used for stereological counting. After delineation of the graft with a $4 \times$ objective, the $\mathrm{TH}$ positive cells with a clearly stained cell body were counted with a $40 \times$ objective. The estimated cell number was calculated by the following equation $N:=(1 / \mathrm{ssf})^{*}(1 / \mathrm{asf})^{*}(1 / \mathrm{hsf})^{*}$ the sum of the counts as described by Kelsen et al. (2010). The section sample fraction (ssf) was 0.3 , because every third section was used for analysis. The area sampling fraction (asf) was set to 1 as $100 \%$ of the graft was analyzed. The height of the sampling fraction (hsf) was 1 as the whole section thickness of $30 \mu \mathrm{m}$ was used. Grafts of controls contained $547 \pm 71 \mathrm{TH}$ positive neurons (mean \pm SEM; $n=6$ ).

\section{Estimation of Remaining TH Positive Fibers in the Striatum}

One section of each rat at the following coordinate in relation to bregma (Paxinos Watson rat brain atlas): anterior $1.5 \mathrm{~mm}$ was selected to determine the remaining $\mathrm{TH}$ positive fiber density in the striatum of rats that did not receive a transplant (second experimental setup), as described previously (Seiler et al., 2016). In brief, the striatum of the lesioned and unlesioned side was photographed by a microscope (Olympus DP72) that was connected to a digital camera (Olympus). The images were converted to 8 bit black and white pictures and inverted using the Fiji software. The mean gray intensity of the dorsal striatum of the lesioned and unlesioned side was measured in a defined area $\left(100,000 \mu \mathrm{m}^{2}\right)$. To account for non-specific background staining, the mean gray value in a defined area $\left(30,000 \mathrm{~mm}^{2}\right)$ in the corpus callosum was measured. The mean gray intensity of the corpus callosum was subtracted from the respective striatal mean gray intensity values and then the values of the lesioned striatum were expressed as percentage of the unlesioned side.

\section{Statistical Analysis}

For statistical analysis a commercially available software package was used (GraphPad Prism 7). To compare group means of several groups repeated two-way ANOVA was used, followed by Tukey's or Bonferroni's multiple comparison test where appropriate. Statistical significance of two groups only, was assessed by two-tailed unpaired $t$-test and the statistical significance levels are expressed as $t \alpha / v$, where $\alpha$ indicates the student's $t$-values and $v$ the relative degree of freedom. Statistical significance was set at $p<0.05$. Data are presented as mean \pm SEM.

\section{RESULTS}

\section{Estimated Extent of the Lesion}

The analysis of TH immunoreactive neurons in the SNc disclosed a lesion extent of $68 \% \pm 3.4 \%$ in the first experimental setup and $36.4 \% \pm 6.0 \%$ in the second. No significant difference between the three levels analyzed or between the saline and NEP1-40 treatment groups was detected.

\section{Behavioral Analyses}

\section{Cylinder Test Experimental Set Up 1}

The asymmetrical forelimb use after unilateral 6-OHDA lesion was assessed in a cylinder. As expected, the lesioned rats 

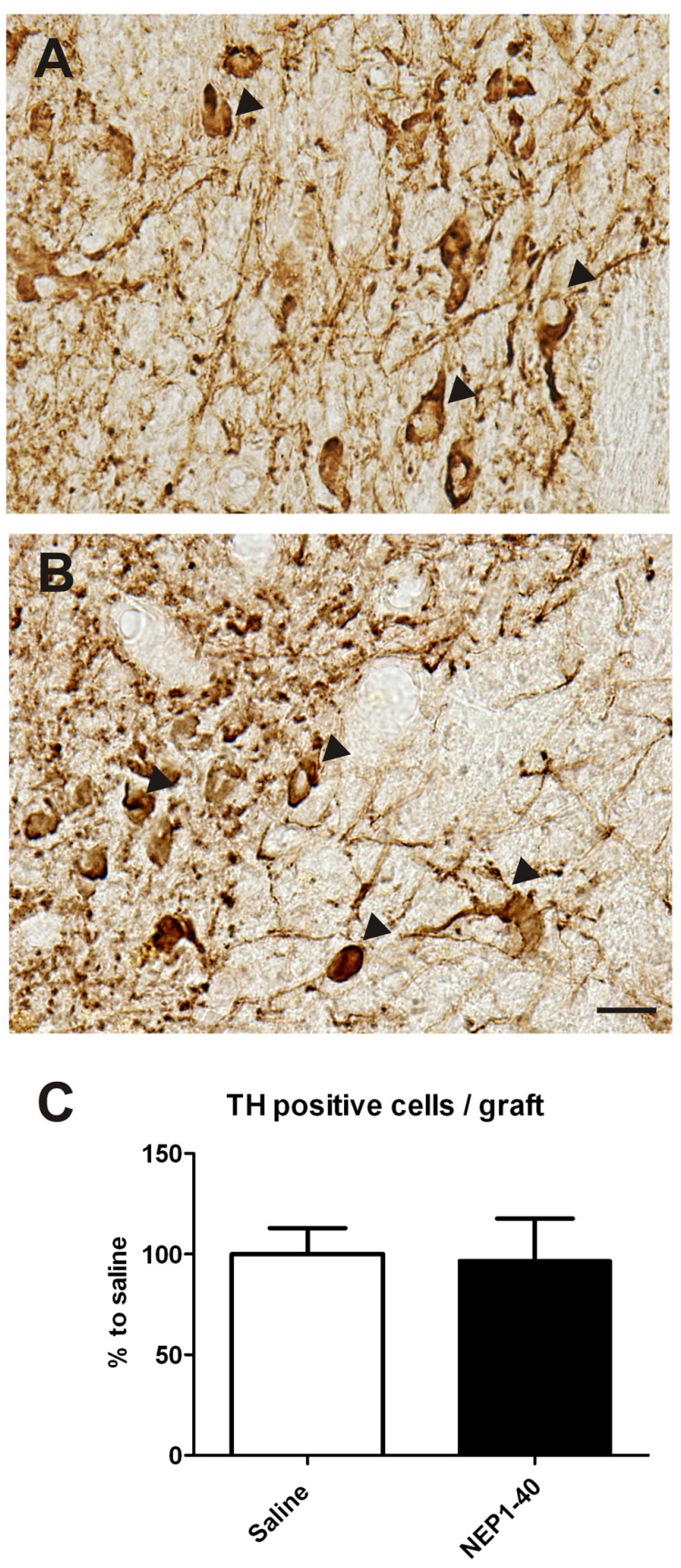

FIGURE 4 | Effects of intra-ventricular infusion of saline or NEP1-40 on tyrosine hydroxylase (TH) positive cell numbers in the grafts.

Representative photomicrographs of TH positive neurons (arrowheads) in the grafts of saline (A) and NEP1-40 treated (B) rats. Scale bar: $20 \mu \mathrm{m}$. No difference between the two groups could be observed, as depicted in the bar graph (C). Data are given as mean + SEM and are presented as percentage of saline.

scored significantly less often with the left paw (contralateral to the lesion) compared to baseline $(49.0 \pm 1.2$ vs. $18.4 \pm 6.0$, $\%$ of left paw use for baseline and lesioned in the saline group, $t_{4.9 / 4} \leq 0.01$ and $50.6 \pm 1.8$ vs. $19.7 \pm 5.2, \%$ of left paw use for baseline and lesioned in the NEP1-40 group, $\left.t_{6.0 / 6} \leq 0.001\right)$ (Figure 2A). No significant difference could be found between NEP1-40 and saline treated groups (data not shown). One week after the transplantation no significant behavioral improvement was observed as compared to the postlesion time point $[26.3 \pm 4.6$ vs. $18.4 \pm 6.0$ and $27.2 \pm 4.8$ vs. $19.7 \pm 5.2$; \% of left paw use for saline and NEP1-40 vs. lesioned, respectively; Time point, $F(2,20)=41.29, p<0.0001$; post hoc, lesioned vs. saline $p=0.33$ and lesioned vs. NEP1$40 p=0.25$ ] (Figure 2A). In contrast, 3 weeks after the transplantation the rats significantly increased the use of the left paw in both treatment groups as compared to the postlesion time point $[32.0 \pm 5.1$ vs. $18.4 \pm 6.0$ and $33.3 \pm 3.1$ vs. $19.7 \pm 5.2$; \% of left paw use for saline and NEP1-40 vs. lesioned, respectively; Time point, $F(2,20)=38.72$, $p<0.0001$; post hoc, lesioned vs. saline $p=0.05$ and lesioned vs. NEP1-40 $p=0.02]$ (Figure 2B). On the other hand, only the NEP140 treated rats showed a significant constant improvement in left paw use over the 5 weeks after transplantation as compared to lesioned $(36.8 \pm 4.8$ vs. $19.7 \pm 5.2 ; \%$ of left paw use for NEP1-40 and lesioned, respectively; Time point, $F(2,20)=28.19, p<0.0001$; post hoc, lesioned vs. NEP1-40 $p=0.01]$ (Figure 2C). In contrast, saline treated rats did not perform significantly better 5 weeks after the transplantation than at the post-lesion time point $[26.2 \pm 5.9$ vs. $18.4 \pm 6.0$; $\%$ of left paw use for saline and lesioned, respectively; Time point, $F(2,20)=28.19, p<0.0001$; post hoc, lesioned vs. saline $p=0.44]$ (Figure 2C). Hence, even though both treatment groups showed a tendency for recovery, at all observed time points all rats performed significantly worse compared to baseline (Figures 2A-C). Moreover, the NEP1-40 treated rats never displayed significantly better results in the behavioral test than the saline treated rats (1Wp.T. $27.2 \pm 4.8$ vs. $26.3 \pm 4.6$; $3 \mathrm{Wp} . \mathrm{T}$. $33.3 \pm 3.1$ vs. $32.0 \pm 5.1$ and 5 Wp.T. $36.8 \pm 4.8$ vs. $26.2 \pm 5.9 ; \%$ of left paw use for NEP1-40 and saline treated rats; Treatment, $F(1,10)=0.07 ; p>0.05$; post hoc, $1 \mathrm{~W}$.T. saline vs. NEP1$40 p=1.0$; Treatment, $F(1,10)=0.12 ; p>0.05$; post hoc, 3 Wp.T. saline vs. NEP1-40 $p=1.0$; Treatment, $F(1,10)=1.01$; $p>0.05$; post hoc, 5Wp.T. saline vs. NEP1-40 $p=0.34]$ (Figures 2A-C).

\section{Cylinder Test Experimental Set Up 2}

The lesioned rats scored significantly less often with the left paw (contralateral to the lesion) compared to baseline $(52.8 \pm 1.6$ vs. $36.4 \pm 3.5$, \% of left paw use for baseline and lesioned in the saline group, $t_{6.6 / 6} \leq 0.01$ and $48.8 \pm 2.2$ vs. $36.7 \pm 5.5$, $\%$ of left paw use for baseline and lesioned in the NEP1-40 group, $t_{3.2 / 6} \leq 0.01$ ) (Figure 2D). No significant difference could be found between NEP1-40 and saline treated groups (data not shown). Five weeks after the transplantation no significant behavioral improvement was observed as compared to the postlesion time point $[42.2 \pm 2.7$ vs. $36.4 \pm 3.5$ and $33.9 \pm 3.4$ vs. $36.7 \pm 5.5$; \% of left paw use for saline and NEP1-40 vs. lesioned, respectively; Time point, $F(2,24)=13.26, p<0.0001$; post hoc, lesioned vs. saline $p=0.38$ and lesioned vs. NEP1-40 $p=0.80]$ (Figure 2D). Moreover, the rats touched the wall with the left paw still significantly less often (Figure 2D). 


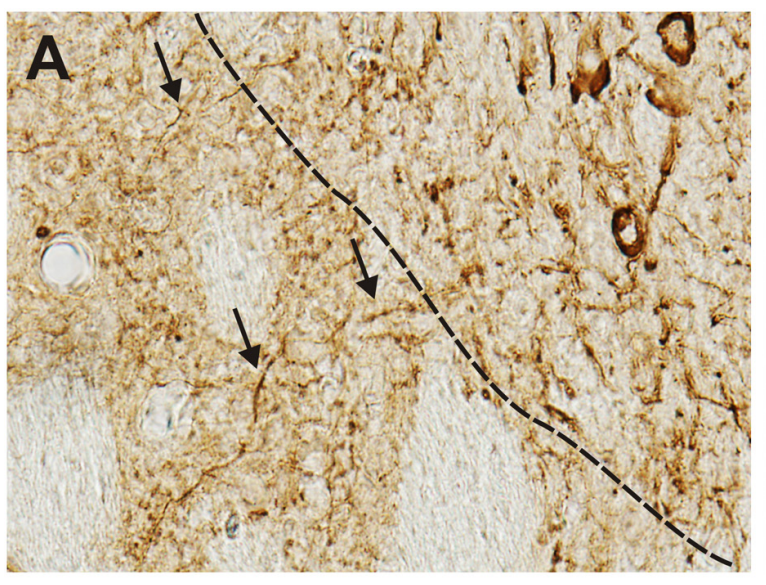

C

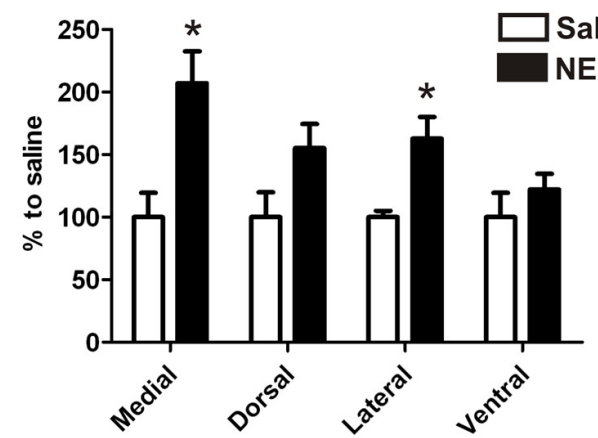

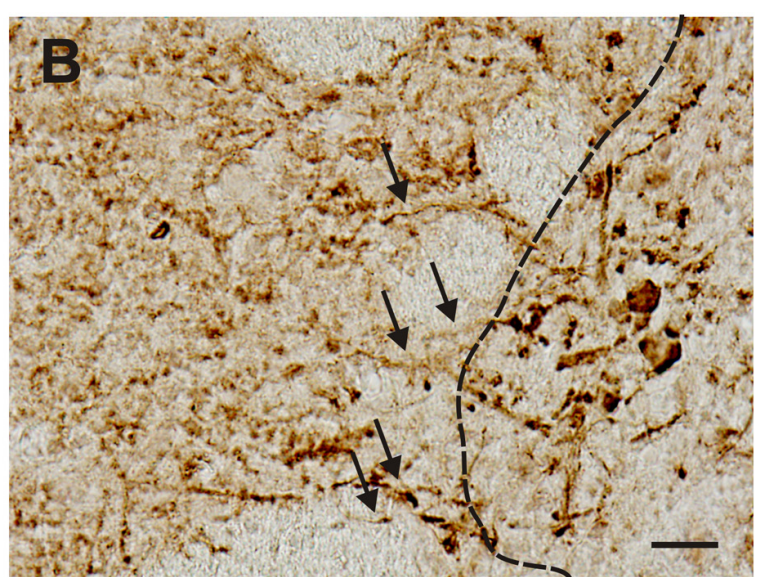

D

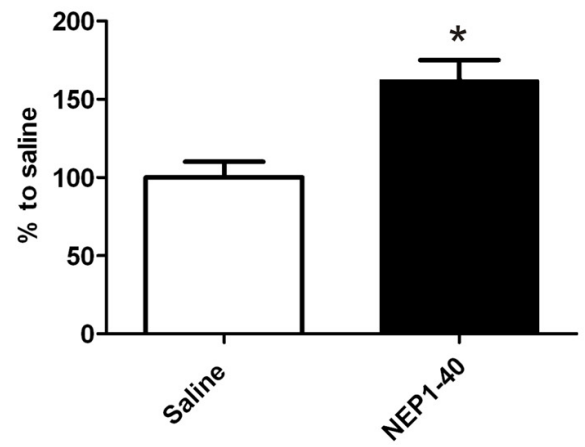

FIGURE 5 | Effects of intra-ventricular infusion of saline or NEP1-40 on tyrosine hydroxylase (TH) positive fiber outgrowth. Representative photomicrographs of TH positive fibers from the graft growing into the host tissue (arrows) from saline (A) and NEP1-40 treated (B) animals. The black dashed line indicates the graft boarder. Note the slight but significant increase in TH positive fibers growing $100 \mu \mathrm{m}$ medially into the host brain resulting in a significant overall larger TH positive fiber outgrowth into the host brain, as indicated in the bar graphs (C,D). Scale bar: $20 \mu \mathrm{m}$. Data are given as mean + SEM and are presented as percentage of corresponding saline. ${ }^{*} p<0.05$.

\section{Histological Analyses}

At the histological level the graft volume did not differ in both treatment groups $(88.6 \% \pm 18.0 \%$ vs. $100 \% \pm 14.7 \%$; for NEP140 and saline treatment, respectively; $t_{0.48 / 11}=0.64$ ) (Figure 3 ). Likewise, the content of surviving $\mathrm{TH}$ positive cells per graft was similar in the two experimental groups $(96.9 \% \pm 20.9 \%$ vs. $100 \% \pm 12.9 \%$; for NEP1-40 and saline treatment; $t_{0.12 / 11}=0.90$ ) (Figure 4). However, NEP1-40 treatment significantly promoted the fiber outgrowth of transplanted $\mathrm{TH}$ positive neurons into the host brain as compared to saline treatment (by 1.6-fold; $\left.t_{3.6 / 11}=0.004\right)$. A more detailed analysis revealed that significantly more $\mathrm{TH}$ positive fibers in the NEP1-40 group grew medially and laterally into the host brain as compared to the saline group $[F(1,11)=13.24 ; p<0.05$; post hoc, $p<0.001$ and $p<0.01$, respectively], whereas dorsal and ventral sites did not show a significant difference between the groups (Figure 5). In experimental setup 2 in which rats were receiving mini osmotic pumps only, no difference in striatal TH positive fiber densities was detected between saline and NEP1-40 treated animals $\left(t_{0.5 / 12}=0.621\right)$ (Figure 6).

\section{DISCUSSION}

This study shows that NgR1 antagonization in combination with tissue grafts significantly promoted $\mathrm{TH}$ positive fiber outgrowth into the host brain of hemi-parkinsonian rats. Moreover, over the post transplantation period the NEP1-40 treated group displayed a tendency for behavioral improvement, however, this did not reach statistical significance. These observations hint to the idea that a certain level of $\mathrm{TH}$ positive fibers growing into the host brain is needed to result in behavioral recovery. In line with this notion, our correlation analyzes for $\mathrm{TH}$ positive neurons in the graft and $\mathrm{TH}$ positive fiber outgrowth with asymmetrical forelimb use showed no association (data not shown). Our results underscore the critical role of the number of surviving $\mathrm{TH}$ positive neurons in the grafts for the improvement of the behavioral deficits within the frame of the experimental conditions described here (Brundin et al., 1988; Meyer et al., 1998). The only partial functional recovery observed in the saline treated control rats grafted with half a VM was expected and purposely chosen to allow monitoring of the efficacy of the NEP1-40 treatment (Meyer et al., 1998; 

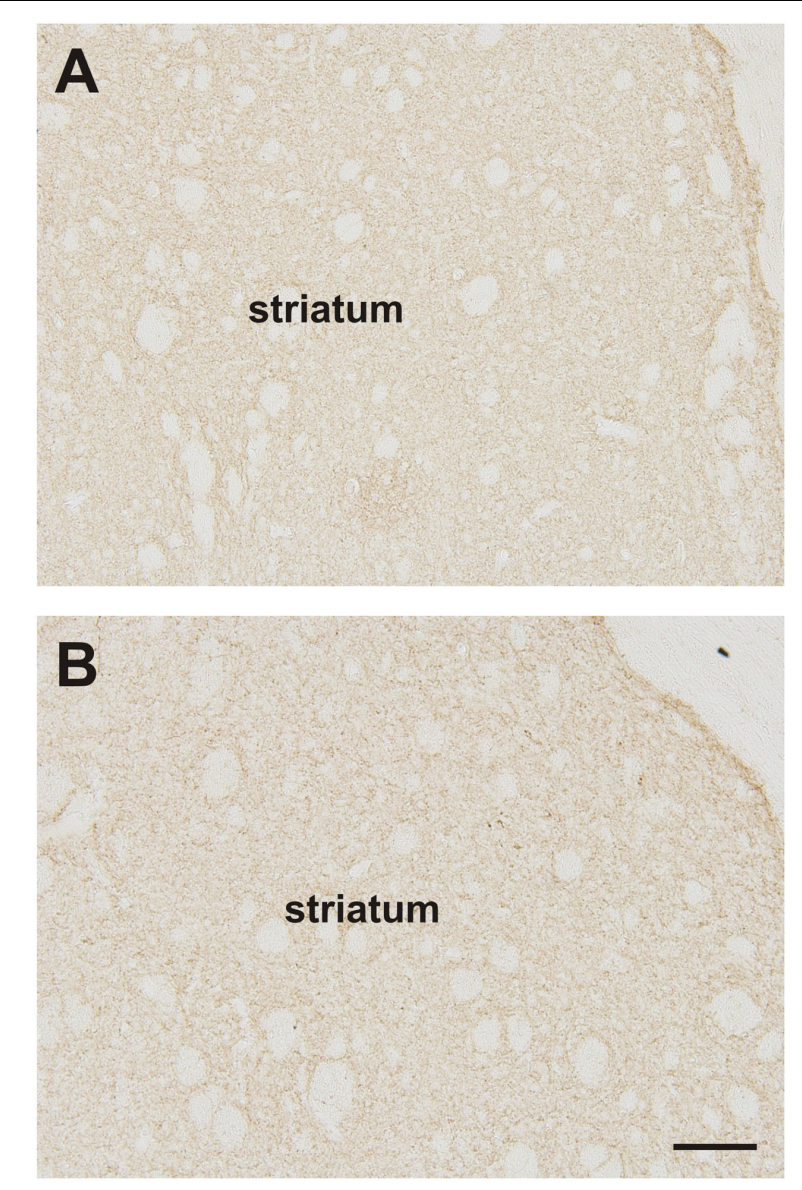

C

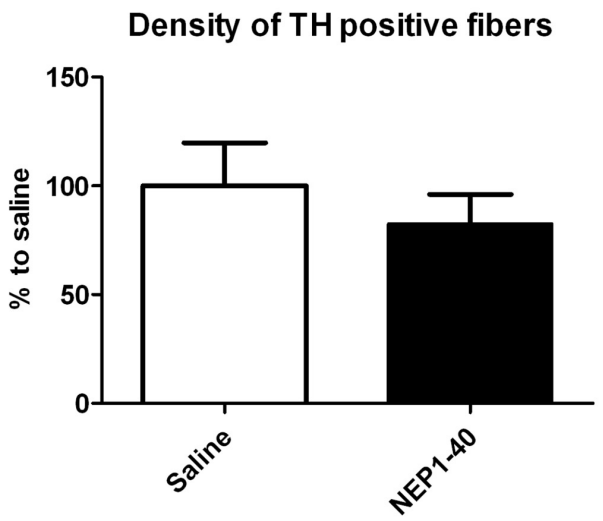

FIGURE 6 | Effects of intra-ventricular infusion of saline or NEP1-40 on striatal tyrosine hydroxylase (TH) positive fiber density. Representative photomicrographs of TH positive fiber densities in the striatum from saline (A) and NEP1-40 (B) treated animals. No difference in striatal TH positive fiber density was detected between the two groups (C). Scale bar: $100 \mu \mathrm{m}$. Data are given as mean + SEM and are presented as percentage of saline.

Seiler et al., 2016). The lack of effect of NEP1-40 treatment on the $\mathrm{TH}$ cell number in the transplants and on the graft volume is, however, in contrast to our previous work, demonstrating an increase in volume and number of $\mathrm{TH}$ positive cells in
VM free-floating roller tube cultures (Seiler et al., 2013). This difference might depend on the actual concentration of NEP140 reaching the VM tissue. While in the culture system the NEP1-40 concentration is defined and stable, NEP1-40 infused into the lateral ventricle is likely diluted in the CSF given the continuous turnover of the CSF (3.4 1/min (Redzic et al., 2005)). NEP1-40 does diffuse from the CSF into the brain parenchyma as several studies have shown (Fang et al., 2010; Wang et al., 2012). The observation that the effects of NEP1-40 treatment on $\mathrm{TH}$ positive fiber outgrowth was most prominent toward the ventricle further support these findings. Nevertheless, the effects of NEP1-40 on VM tissue grafts in our study might have been underestimated, even though we have used a concentration as previously described (Wang et al., 2012), and further studies using higher concentrations of NEP1-40 are needed. Given that in our experimental model the striatum is not completely denervated by 6-OHDA and NEP1-40 penetrates into the parenchyma, we next reasoned whether NEP1-40 might target the spared $\mathrm{TH}$ fibers. In experimental setup 2 we tested for that hypothesis. In our measurements of $\mathrm{TH}$ positive fiber densities, however, we found no evidence that NEP1-40 exerts effect on TH fiber densities in absence of a graft. Importantly to note, we cannot exclude that NEP1-40 treatment exerts other effects on the brain, such as for example on microglia (Fang et al., 2015).

These results complement our previous findings gathered using the same experimental setup investigating the potential of inhibition of Nogo-A/NgR1 signaling by means of ventricular infusion of neutralizing Nogo-A antibodies (Seiler et al., 2016). So, we reported that anti-Nogo-A antibody treated rats gradually improved their performance in the cylinder test, reaching almost baseline levels 5 weeks after the transplantation (Seiler et al., 2016). In this respect it is important to note that NEP1-40 treatment seems to have a substantially lower capacity to support graft function. We hypothesize that the different signaling partners and mechanisms activated by Nogo-neutralization and by its receptor antagonization might account, at least in part, for the dissimilar effects observed in the two distinct studies. In fact, the neutralizing anti-Nogo-A antibodies used in the previous work targeted the $\Delta-20$ domain of Nogo-A, which is known to bind to the sphingosine-1 phosphate receptor 2 (S1PR2) but not to the NgR1 (Akbik et al., 2012; Kurowska et al., 2014; Saha et al., 2014). Hence, functional recovery and increased $\mathrm{TH}$ positive fiber outgrowth after anti-Nogo-A antibody infusion in our previous study was observed even though the Nogo-A-NgR1 pathway was still active. Given that the NgR1 does not only bind Nogo-A through its Nogo66 domain, but also other growth inhibitors such as myelin associated glycoprotein, the oligodendrocyte myelin glycoprotein and the chondroitin sulfate proteoglycans (Akbik et al., 2012; Schwab and Strittmatter, 2014), it was tempting to speculate that antagonization of the NgR1 might be more potent in enhancing graft function and $\mathrm{TH}$ positive fiber outgrowth than inhibiting specifically Nogo-A. The results of the present study, however, did not support this notion even though the above mentioned issues need to be addressed in more depth to draw a final conclusion. 


\section{CONCLUSION}

In sum, the present study demonstrates that NEP1-40 treatment of hemi-parkinsonian rats promoted $\mathrm{TH}$ positive fiber outgrowth into the host brain which, however, was not sufficient to induce functional recovery to baseline levels. In line with the latter observation NEP1-40 treatment did not enhance graft volume or survival of transplanted dopaminergic neurons. Alternative strategies to inhibit Nogo-A signaling as for example through neutralizing anti-Nogo-A antibodies possibly in combination with application of neurotrophic factors should be considered to enhance engraftment and function of the transplant.

\section{AUTHOR CONTRIBUTIONS}

Author's contribution to the study and manuscript preparation includes the following. Conception and design of the work was done by SS, SD, and HW. Acquisition of data was performed by

\section{REFERENCES}

Akbik, F., Cafferty, W. B., and Strittmatter, S. M. (2012). Myelin associated inhibitors: a link between injury-induced and experience-dependent plasticity. Exp. Neurol. 235, 43-52. doi: 10.1016/j.expneurol.2011.06.006

Alsberg, E., von Recum, H. A., and Mahoney, M. J. (2006). Environmental cues to guide stem cell fate decision for tissue engineering applications. Expert Opin. Biol. Ther. 6, 847-866. doi: 10.1517/14712598.6.9.847

Andereggen, L., Meyer, M., Guzman, R., Ducray, A. D., and Widmer, H. R. (2009). Effects of GDNF pretreatment on function and survival of transplanted fetal ventral mesencephalic cells in the 6-OHDA rat model of Parkinson's disease. Brain Res. 1276, 39-49. doi: 10.1016/j.brainres.2009.04.021

Bjorklund, A., Dunnett, S. B., Brundin, P., Stoessl, A. J., Freed, C. R., Breeze, R. E., et al. (2003). Neural transplantation for the treatment of Parkinson's disease. Lancet Neurol. 2, 437-445. doi: 10.1016/S1474-4422(03)00442-3

Boix, J., Padel, T., and Paul, G. (2015). A partial lesion model of Parkinson's disease in mice-characterization of a 6-OHDA-induced medial forebrain bundle lesion. Behav. Brain Res. 284, 196-206. doi: 10.1016/j.bbr.2015.01.053

Brooks, S. P., and Dunnett, S. B. (2009). Tests to assess motor phenotype in mice: a user's guide. Nat. Rev. Neurosci. 10, 519-529. doi: 10.1038/nrn2652

Brundin, P., Barbin, G., Strecker, R. E., Isacson, O., Prochiantz, A., and Bjorklund, A. (1988). Survival and function of dissociated rat dopamine neurones grafted at different developmental stages or after being cultured in vitro. Brain Res. 467, 233-243. doi: 10.1016/0165-3806(88)90027-2

Chaturvedi, R. K., Shukla, S., Seth, K., and Agrawal, A. K. (2006). Nerve growth factor increases survival of dopaminergic graft, rescue nigral dopaminergic neurons and restores functional deficits in rat model of Parkinson's disease. Neurosci. Lett. 398, 44-49. doi: 10.1016/j.neulet.2005.12.042

Emgard, M., Karlsson, J., Hansson, O., and Brundin, P. (1999). Patterns of cell death and dopaminergic neuron survival in intrastriatal nigral grafts. Exp. Neurol. 160, 279-288. doi: 10.1006/exnr.1999.7198

Espejo, M., Cutillas, B., Arenas, T. E., and Ambrosio, S. (2000). Increased survival of dopaminergic neurons in striatal grafts of fetal ventral mesencephalic cells exposed to neurotrophin-3 or glial cell line-derived neurotrophic factor. Cell Transplant. 9, 45-53.

Fang, P. C., Barbay, S., Plautz, E. J., Hoover, E., Strittmatter, S. M., and Nudo, R. J. (2010). Combination of NEP 1-40 treatment and motor training enhances behavioral recovery after a focal cortical infarct in rats. Stroke 41, 544-549. doi: 10.1161/STROKEAHA.109.572073

Fang, Y., Yan, J., Li, C., Zhou, X., Yao, L., Pang, T., et al. (2015). The Nogo/Nogo receptor $(\mathrm{NgR})$ signal is involved in neuroinflammation through the regulation of microglial inflammatory activation. J. Biol. Chem. 290, 28901-28914. doi: 10.1074/jbc.M115.678326
SS, SD, LA, and HW. Analysis and interpretation of data was carried out by SS, SD, LA, and HW. The draft of the article was performed by SS and critically revised by SD and HW. All authors read and approved the final manuscript.

\section{FUNDING}

This research was supported by the Swiss Parkinson Foundation, the HANELA Foundation and the Swiss National Science Foundation (No. 31003A_135565).

\section{ACKNOWLEDGMENTS}

The expert technical assistance from Susanne Wälchli is gratefully acknowledged. Microscopy was performed on equipment supported by the Microscopy Imaging Center (MIC), University of Bern, Switzerland.

Gonzenbach, R. R., and Schwab, M. E. (2008). Disinhibition of neurite growth to repair the injured adult CNS: focusing on Nogo. Cell. Mol. Life Sci. 65, 161-176. doi: 10.1007/s00018-007-7170-3

GrandPre, T., Li, S., and Strittmatter, S. M. (2002). Nogo-66 receptor antagonist peptide promotes axonal regeneration. Nature 417, 547-551. doi: 10.1038/ $417547 \mathrm{a}$

Hallett, P. J., Cooper, O., Sadi, D., Robertson, H., Mendez, I., and Isacson, O. (2014). Long-term health of dopaminergic neuron transplants in Parkinson's disease patients. Cell Rep. 7, 1755-1761. doi: 10.1016/j.celrep.2014.05.027

Haque, N. S., Hlavin, M. L., Fawcett, J. W., and Dunnett, S. B. (1996). The neurotrophin NT4/5, but not NT3, enhances the efficacy of nigral grafts in a rat model of Parkinson's disease. Brain Res. 712, 45-52. doi: 10.1016/0006-8993(95) 01427-6

Inoue, H., Lin, L., Lee, X., Shao, Z., Mendes, S., Snodgrass-Belt, P., et al. (2007). Inhibition of the leucine-rich repeat protein LINGO-1 enhances survival, structure, and function of dopaminergic neurons in Parkinson's disease models. Proc. Natl. Acad. Sci. U.S.A. 104, 14430-14435. doi: 10.1073/pnas.0700 901104

Karlsson, J., Emgard, M., Gido, G., Wieloch, T., and Brundin, P. (2000). Increased survival of embryonic nigral neurons when grafted to hypothermic rats. Neuroreport 11, 1665-1668. doi: 10.1097/00001756-200006050-00014

Kefalopoulou, Z., Politis, M., Piccini, P., Mencacci, N., Bhatia, K., Jahanshahi, M., et al. (2014). Long-term clinical outcome of fetal cell transplantation for Parkinson disease: two case reports. JAMA Neurol. 71, 83-87. doi: 10.1001/ jamaneurol.2013.4749

Kelsen, J., Larsen, M. H., Sorensen, J. C., Moller, A., Frokiaer, J., Nielsen, S., et al. (2010). Neuronal precursor cell proliferation in the hippocampus after transient cerebral ischemia: a comparative study of two rat strains using stereological tools. Exp. Transl. Stroke M ed. 2:8. doi: 10.1186/2040-7378-2-8

Kurowska, Z., Brundin, P., Schwab, M. E., and Li, J. Y. (2014). Intracellular Nogo-A facilitates initiation of neurite formation in mouse midbrain neurons in vitro. Neuroscience 256, 456-466. doi: 10.1016/j.neuroscience.2013.10.029

Meyer, M., Widmer, H. R., Wagner, B., Guzman, R., Evtouchenko, L., Seiler, R. W., et al. (1998). Comparison of mesencephalic free-floating tissue culture grafts and cell suspension grafts in the 6 hydroxydopamine-lesioned rat. Exp. Brain Res. 119, 345-355. doi: 10.1007/s002210050350

Ourednik, V., and Ourednik, J. (2005). Graft/host relationships in the developing and regenerating CNS of mammals. Ann. N. Y. Acad. Sci. 1049, 172-184. doi: 10.1196/annals.1334.016

Pantcheva, P., Reyes, S., Hoover, J., Kaelber, S., and Borlongan, C. V. (2015). Treating non-motor symptoms of Parkinson's disease with transplantation of stem cells. Expert Rev. Neurother. 15, 1231-1240. doi: 10.1586/14737175.2015. 1091727 
Piquet, A. L., Venkiteswaran, K., Marupudi, N. I., Berk, M., and Subramanian, T. (2012). The immunological challenges of cell transplantation for the treatment of Parkinson's disease. Brain Res. Bull. 88, 320-331. doi: 10.1016/j.brainresbull. 2012.03.001

Rath, A., Klein, A., Papazoglou, A., Pruszak, J., Garcia, J., Krause, M., et al. (2013). Survival and functional restoration of human fetal ventral mesencephalon following transplantation in a rat model of Parkinson's disease. Cell Transplant. 22, 1281-1293. doi: 10.3727/096368912X654984

Redzic, Z. B., Preston, J. E., Duncan, J. A., Chodobski, A., and SzmydyngerChodobska, J. (2005). The choroid plexus-cerebrospinal fluid system: from development to aging. Curr. Top. Dev. Biol. 71, 1-52. doi: 10.1016/S00702153(05)71001-2

Rosenblad, C., Martinez-Serrano, A., and Bjorklund, A. (1996). Glial cell line-derived neurotrophic factor increases survival, growth and function of intrastriatal fetal nigral dopaminergic grafts. Neuroscience 75, 979-985.

Saha, N., Kolev, M., and Nikolov, D. B. (2014). Structural features of the Nogo receptor signaling complexes at the neuron/myelin interface. Neurosci. Res. 87, 1-7. doi: 10.1016/j.neures.2014.06.003

Schaar, K. L., Brenneman, M. M., and Savitz, S. I. (2010). Functional assessments in the rodent stroke model. Exp. Transl. Stroke Med. 2:13. doi: 10.1186/20407378-2-13

Schmandke, A., Schmandke, A., and Schwab, M. E. (2014). Nogo-A: multiple roles in CNS development, maintenance, and disease. Neuroscientist 20, 372-386. doi: $10.1177 / 1073858413516800$

Schwab, M. E., and Strittmatter, S. M. (2014). Nogo limits neural plasticity and recovery from injury. Curr. Opin. Neurobiol. 27, 53-60. doi: 10.1016/j.conb. 2014.02.011

Seiler, S., Di Santo, S., and Widmer, H. R. (2016). Nogo-A neutralization improves graft function in a rat model of Parkinson's disease. Front. Cell. Neurosci. 10:87. doi: 10.3389/fncel.2016.00087

Seiler, S., Pollini, D., Di Santo, S., and Widmer, H. R. (2013). Antagonizing Nogo-receptor 1 promotes the number of cultured dopaminergic neurons and elongates their neurites. Neuroreport 24, 1047-1052. doi: 10.1097/WNR. 0000000000000063

Sinclair, S. R., Svendsen, C. N., Torres, E. M., Martin, D., Fawcett, J. W., and Dunnett, S. B. (1996). GDNF enhances dopaminergic cell survival and fibre outgrowth in embryonic nigral grafts. Neuroreport 7, 2547-2552. doi: 10.1097/ 00001756-199611040-00029

Spenger, C., Studer, L., Evtouchenko, L., Egli, M., Burgunder, J. M., Markwalder, R., et al. (1994). Long-term survival of dopaminergic neurones in free-floating roller tube cultures of human fetal ventral mesencephalon. J. Neurosci. Methods 54, 63-73. doi: 10.1016/0165-0270(94)90160-0
Stefanova, N., Bucke, P., Duerr, S., and Wenning, G. K. (2009). Multiple system atrophy: an update. Lancet Neurol. 8, 1172-1178. doi: 10.1016/S1474-4422(09) 70288-1

Tatard, V. M., Sindji, L., Branton, J. G., Aubert-Pouessel, A., Colleau, J., Benoit, J. P., et al. (2007). Pharmacologically active microcarriers releasing glial cell line - derived neurotrophic factor: survival and differentiation of embryonic dopaminergic neurons after grafting in hemiparkinsonian rats. Biomaterials 28, 1978-1988. doi: 10.1016/j.biomaterials.2006.12.021

Thompson, L. H., Grealish, S., Kirik, D., and Bjorklund, A. (2009). Reconstruction of the nigrostriatal dopamine pathway in the adult mouse brain. Eur. J. Neurosci. 30, 625-638. doi: 10.1111/j.1460-9568.2009.06878.x

Tronci, E., Shin, E., Bjorklund, A., and Carta, M. (2012). Amphetamine-induced rotation and L-DOPA-induced dyskinesia in the rat 6-OHDA model: a correlation study. Neurosci. Res. 73, 168-172. doi: 10.1016/j.neures.2012.03.004

Wang, F., Xing, S., He, M., Hou, Q., Chen, S., Zou, X., et al. (2012). Nogo-A is associated with secondary degeneration of substantia nigra in hypertensive rats with focal cortical infarction. Brain Res. 1469, 153-163. doi: 10.1016/j.brainres. 2012.06.040

West, M. J., Slomianka, L., and Gundersen, H. J. (1991). Unbiased stereological estimation of the total number of neurons in thesubdivisions of the rat hippocampus using the optical fractionator. Anat. Rec. 231, 482-497. doi: 10.1002/ar.1092310411

Yasuhara, T., Matsukawa, N., Hara, K., Yu, G., Xu, L., Maki, M., et al. (2006). Transplantation of human neural stem cells exerts neuroprotection in a rat model of Parkinson's disease. J. Neurosci. 26, 12497-12511. doi: 10.1523/ JNEUROSCI.3719-06.2006

Yurek, D. M. (1998). Glial cell line-derived neurotrophic factor improves survival of dopaminergic neurons in transplants of fetal ventral mesencephalic tissue. Exp. Neurol. 153, 195-202. doi: 10.1006/exnr.1998.6884

Yurek, D. M., Lu, W., Hipkens, S., and Wiegand, S. J. (1996). BDNF enhances the functional reinnervation of the striatum by grafted fetal dopamine neurons. Exp. Neurol. 137, 105-118. doi: 10.1006/exnr.1996.0011

Conflict of Interest Statement: The authors declare that the research was conducted in the absence of any commercial or financial relationships that could be construed as a potential conflict of interest.

Copyright (C) 2017 Seiler, Di Santo, Andereggen and Widmer. This is an open-access article distributed under the terms of the Creative Commons Attribution License (CC BY). The use, distribution or reproduction in other forums is permitted, provided the original author(s) or licensor are credited and that the original publication in this journal is cited, in accordance with accepted academic practice. No use, distribution or reproduction is permitted which does not comply with these terms. 